\title{
Does Simple Steatosis Affect Liver Regeneration after Partial Hepatectomy in Rats?
}

\author{
Tomáš Garnol, Otto Kučera*, Pavla Staňková, Halka Lotková, Zuzana Červinková
}

\author{
Department of Physiology, Charles University in Prague, Faculty of Medicine in Hradec Králové, Hradec Králové, Czech \\ Republic \\ * Corresponding author: Charles University in Prague, Faculty of Medicine in Hradec Králové, Šimkova 870, 50038 Hradec \\ Králové 1, Czech Republic; e-mail: kucerao@1fhk.cuni.cz
}

\begin{abstract}
Summary: Aim: The aim of our study was to assess whether simple steatosis impairs liver regeneration after partial hepatectomy (PHx) in rats. Methods: Male Sprague-Dawley rats were fed a standard diet (ST-1, 10\% kcal fat) and high-fat diet (HFD, 71\% kcal fat) for 6 weeks. Then the rats were submitted to 2/3 PHx and animals were sacrificed 24, 48 or $72 \mathrm{~h}$ after PHx. Serum biochemistry, respiration of mitochondria in liver homogenate, hepatic oxidative stress markers, selected cytokines and DNA content were measured, and histopathological samples were prepared. Liver regeneration was evaluated by incorporation of bromodeoxyuridine (BrdU) to hepatocyte DNA. Results: HFD induced simple microvesicular liver steatosis. PHx caused elevation of serum markers of liver injury in both groups; however, an increase in these parameters was delayed in HFD group. Hepatic content of reduced glutathione was significantly increased in both groups after PHx. There were no significant changes in activities of respiratory complexes I and II (state 3). Relative and absolute liver weights, total DNA content, and DNA synthesis exerted very similar changes in both ST-1 and HFD groups after PHx. Conclusion: PHx-induced regeneration of the rat liver with simple steatosis was not significantly affected when compared to the lean liver.
\end{abstract}

Keywords: Fatty liver; Liver regeneration; Partial hepatectomy

\section{Introduction}

The liver has a unique and remarkable capacity for self-renewal after its damage. This regenerative potential is essential for survival after liver injury induced by toxic substances, viral infections, metabolic and immune dysfunctions and in response to surgical removal of a part of liver tissue $(1,2)$. Liver regeneration is most commonly studied by performing partial hepatectomy $(\mathrm{PHx})$; the classical model represents removal of $2 / 3$ of the liver mass in rodents as described by Higgins and Anderson (3). Advantage of PHx model of liver regeneration in comparison with other regenerative stimuli is the absence of injury to the remnant of liver tissue after surgery. Liver regeneration after partial hepatectomy is a very complex and well-orchestrated process associated with signaling cascades involving growth factors, cytokines, matrix remodeling, several feedbacks of stimulation and inhibition of growth related signals, and metabolic changes (4-6). Nevertheless, the fully integrated understanding of the mechanisms involved in the regulation of liver regeneration remains to be elucidated. The signals responsible for initiation and especially termination of liver regeneration are not completely defined yet (7). The interest to understand liver regeneration pushes not only ambition to understand this unique process of well controlled tissue proliferation but also clinical practice. Resection of liver for different reasons (primary and metastatic tumors, living donor of liver grafts for transplantation, etc.) has become a common clinical practice. The success of recovery and renewal of liver functions depends on the regeneration of the liver remnant. Pre-existing pathological abnormalities among which hepatic steatosis is one of the most frequent disorders may significantly deteriorate the course of liver regeneration after surgical resection and regeneration of steatotic graft after liver transplantation $(8,9)$.

Non-alcoholic fatty liver disease (NAFLD) is the most frequent hepatic disorder in the Western countries and its prevalence is still increasing (10). NAFLD refers to a wide spectrum of liver damages, ranging from simple steatosis to non-alcoholic steatohepatitis (NASH), advanced fibrosis, and cirrhosis. As described by Chalasani and coworkers the definition of NAFLD requires that firstly there is evidence of hepatic steatosis, either by imaging or by histology and secondly there are no causes for secondary hepatic fat accumulation such as significant alcohol consumption, use of steatogenic medication, or hereditary disorders (11).

NAFLD is associated with mitochondrial oxidative alterations (12), increased production of reactive oxygen and nitrogen species and decreased liver content of reduced glutathione $(\mathrm{GSH})(13,14)$. Accumulating evidence indi- 
cates that mitochondrial dysfunction participates as a key player in the pathophysiology of NAFLD (15). Besides ultrastructural changes (16) and depletion of mitochondrial DNA $(17,18)$, mitochondrial dysfunction includes altered activities of respiratory complexes and decreased capacity for ATP synthesis $(19,20)$. Complex I (NADH:ubiquinone oxidoreductase) and complex II (succinate-ubiquinone oxidoreductase) are two respiratory complexes through which electrons enter respiratory chain. Their damage affects mitochondrial energy production and moreover dysfunction of complex I leads to considerable generation of reactive oxygen species (ROS).

There are several studies focused on regeneration of the liver affected by NAFLD with inconsistent results. There is evidence about impaired functional recovery and hepatocellular regeneration of the liver affected by steatosis after PHx in rats $(21,22)$. Similar results were found also in humans. Hepatic steatosis impairs liver regeneration as is reflected by the declining regeneration markers in patients with an increasing degree of steatosis (23). Patients with steatosis had an up to two-fold increased risk of postoperative complications, and those with excessive steatosis had an almost threefold increased risk of death after major hepatic resection (24). On the contrary other authors did not see impaired regenerative response of steatotic liver in rats after partial hepatectomy $(25,26)$. Cho and coworkers document that mild hepatic steatosis is not a major risk factor for hepatectomy, and that regenerative power is not impaired in living liver donors (27). To clarify the ability of steatotic liver to regenerate is important for clinical practice, e.g. for decision if the liver affected by NAFLD could be used for transplantation. The aim of our study was to evaluate whether simple steatosis affects the early course of liver regeneration after PHx. Important limiting step of liver regeneration is sufficient energy availability. Therefore we decided to assess in addition to the markers of liver injury and regeneration also oxygen uptake by mitochondria in liver homogenates.

\section{Materials and Methods}

\section{Experimental design}

Male Sprague-Dawley rats (AnLab, Prague, Czech Republic) with initial body weight of $240 \pm 20 \mathrm{~g}$ were used throughout the study. The rats were housed at $22 \pm 2{ }^{\circ} \mathrm{C}, 55$ $\pm 10 \%$ humidity, air exchange 10 times/h and $12 \mathrm{~h}$ light-dark cycle. The animals had free access to tap water and different diets as described below. In accordance with Czech legislation, all animals received care according to the guidelines set out by the Animal-Welfare Body of the Charles University, Prague, Czech Republic, and the International Guiding Principles for Biomedical Research Involving Animals and our study was approved by this committee and by the Ministry of Education, Youth and Sports (MSMT 18324/2008-30).

Rats were fed ad libitum a standard pelleted diet (DOS 2B, Velaz, Prague, Czech Republic; 10\% energy fat, 30\% energy proteins, $60 \%$ energy saccharides, ST-1) or highfat diet (HFD; $71 \%$ energy fat, $18 \%$ energy proteins, $11 \%$ energy saccharides) according to Lieber (28) modified by Kučera (14) for 6 weeks. Then the animals were submitted to $2 / 3$ partial hepatectomy $(n=6$ in each group) $(65-70 \%$ of the liver tissue was removed comprising left lateral and median lobules of the liver) described by Higgins and Anderson (3) or laparotomy (sham operation, LAP, $n=4$ ). Animals were sacrificed 24, 48 or 72 hours after PHx or LAP by exsanguination from aortic bifurcation and liver samples were collected for analyses. High-fat diet was prepared from ingredients purchased from MP Biomedicals (Solon, $\mathrm{OH}$, USA). All chemicals, unless otherwise mentioned, were of analytical grade and were obtained from Sigma-Aldrich. After starvation for $14 \mathrm{~h}$, the animals were sacrificed and liver and serum samples were taken. Samples for consequent evaluation were immediately frozen in liquid nitrogen and stored at $-80{ }^{\circ} \mathrm{C}$ until analysis.

\section{Serum biochemical measurements}

Serum concentrations of total bilirubin, and activities of alanine aminotransferase (ALT), aspartate aminotransferase (AST) and alkaline phosphatase (ALP) were determined by routine laboratory methods (commercial sets, Roche Diagnostics) on Cobas Integra 800 (Roche Diagnostics) in the Institute for Clinical Biochemistry and Diagnostics, University Hospital in Hradec Králové.

\section{Measurement of oxygen uptake by mitochondria in liver homogenate}

Rat liver tissue was homogenized as previously described and oxygen consumption in liver homogenate $(n=3-4)$ was measured by a high-resolution respirometry using Oxygraph $2 \mathrm{k}$ (Oroboros Instruments, Innsbruck, Austria) $(29,30)$. The rate of oxygen consumption was evaluated using Oroboros DatLab 4 software and expressed as nmol oxygen/s/mg protein. Respiratory control index (RCI) for complex II substrates was calculated.

\section{Determination of glutathione}

Liver homogenate was added into cold 10\% metaphosphoric acid, shaken and centrifuged $(20,000 \times \mathrm{g}, 10 \mathrm{~min}$, $4{ }^{\circ} \mathrm{C}$ ). Glutathione in the supernatant was analyzed by a modified fluorimetric method $(31,32)$. Briefly, reduced (GSH) an oxidized (GSSG) glutathione was allowed to react with o-phthalaldehyde in phosphate buffer, and the fluorimetric detection was carried out $\left(\lambda_{\mathrm{Ex}}=340 \mathrm{~nm}, \lambda_{\mathrm{Em}}=420 \mathrm{~nm}\right)$.

\section{Determination of tissue triacylglycerols, cholesterol and DNA}

Lipids from rat livers were prepared using chloroform-methanol extraction (33). Total cholesterol and 
triacylglycerols (TAG) were measured using commercial kits (Roche Diagnostics GmbH, Mannheim, Germany). DNA was determined by means of the diphenylamine reagent according to Burton (34).

\section{Determination of serum and liver tissue cytokines and hepatic malondialdehyde}

Liver samples were homogenized in RIPA buffer, centrifuged $(10,000 \times \mathrm{g})$ and the supernatant was collected. Protein content in the sample was determined by the method of Bradford (35) using bovine serum albumin as a standard. Concentrations of liver interleukin-6 (IL-6) and transforming growth factor- $\beta 1$ (TGF- $\beta 1$ ) in the supernatant were measured by enzyme linked immunosorbent assay (ELISA) (BMS625, BMS623, Bender MedSystems, Vienna, Austria). Total tissue malondialdehyde (MDA) was analyzed using a slightly modified method of Pilz (36). Briefly, derivatization with 2,4-dinitrophenylhydrazine was performed after an alkaline hydrolysis, and subsequent reversed-phase high-performance liquid chromatography (Agilent, Palo Alto, CA, USA).

\section{Liver histology, bromodeoxyuridine staining and its quantification}

Liver samples were taken immediately after the rats were sacrificed and fixed by immersion in 4\% neutral formaldehyde. Paraffin sections were stained with hematoxylin $\&$ eosin. Fat accumulation in hepatocytes was confirmed by staining of formaldehyde-fixed frozen liver sections with oil red.

The immunohistochemical analysis of bromodeoxyuridine(BrdU)-stained samples was performed on paraffin sections of liver tissue ( $6 \mu \mathrm{m}$ thick) as described previously (37). Quantification of BrdU-positive nuclei was performed in at least 10 microscope fields $(10 \times$ objective magnification) in each section using NIS-Elements AR 2.30 (Nikon, Lewisville, TX).

\section{Statistical analysis}

The results are expressed as the mean \pm SD. Analyses were performed using Graph-Pad Prism 4.03 software (Graph Pad Software, San Diego, CA, USA). First, normality was tested by Kolmogorov-Smirnov test. In normal data, comparisons were made among the groups using ANOVA followed by Tukey-Kramer's post hoc test. In the case of non-Gaussian distribution, non-parametric Kruskal-Wallis test and Dunn's post hoc test were used. $\mathrm{P}<0.05$ was considered statistically significant. Because there were not significant differences between control non-operated groups and laparotomized groups in either ST-1 or HFD feedings, respectively, we do not present results of sham-operated groups.

\section{Results}

\section{Serum characteristics}

As shown in table 1, PHx induced mild injury to the liver as documented by increased activities of ALT ( $24 \mathrm{~h}$, $\mathrm{p}<0.001)$, AST (24 and $48 \mathrm{~h}, \mathrm{p}<0.001$ and 0.05 , respectively) and ALP (24 and $48 \mathrm{~h}, \mathrm{p}<0.05)$ in ST-1 rats. In HFD animals, elevation of markers of liver injury was significantly delayed when compared to ST-1 (ALT $72 \mathrm{~h}, \mathrm{p}<0.05$; ALP 48 and $72 \mathrm{~h}, \mathrm{p}<0.05$ and 0.001 , respectively; total bilirubin concentration $72 \mathrm{~h}, \mathrm{p}<0.05)$.

\section{Histological parameters}

Livers of rats fed by HFD showed simple microvesicular steatosis without inflammatory infiltrate, hepatocyte necrosis or fibrosis (data not shown). PHx induced regenerative response of hepatocytes which was visualized by staining of the incorporation of BrdU in hepatocyte DNA (Fig 1A-D). Semiquantitative analysis of BrdU-positive nuclei (Fig. 2) showed a peak DNA synthesis 24 hours after PHx in both

Tab. 1: Basal serum characteristics of the groups.

\begin{tabular}{|l|c|c|c|c|c|c|c|c|}
\hline $\begin{array}{l}\text { Serum } \\
\text { characteristics }\end{array}$ & ST-1 & $\begin{array}{c}\text { ST-1_PHx } \\
\mathbf{2 4 h}\end{array}$ & $\begin{array}{c}\text { ST-1_PHx } \\
\mathbf{4 8} \mathbf{h}\end{array}$ & $\begin{array}{c}\text { ST-1_PHx } \\
\mathbf{7 2} \mathbf{h}\end{array}$ & HFD & $\begin{array}{c}\text { HFD_PHx } \\
\mathbf{2 4 h}\end{array}$ & $\begin{array}{c}\text { HFD_PHx } \\
\mathbf{4 8 h}\end{array}$ & $\begin{array}{c}\text { HFD_PHx } \\
\mathbf{7 2 h}\end{array}$ \\
\hline ALT $(\mu \mathrm{kat} / \mathrm{l})$ & $0.7 \pm 0.1$ & $\mathbf{4 . 6} \pm \mathbf{3 . 3 * *}$ & $2.2 \pm 1.9$ & $1.4 \pm 0.4$ & $0.9 \pm 0.3$ & $1.8 \pm 0.5$ & $1.2 \pm 0.4$ & $\mathbf{2 . 5} \pm \mathbf{1 . 1}^{\#}$ \\
\hline AST $(\mu \mathrm{kat} / \mathrm{l})$ & $1.9 \pm 0.2$ & $\mathbf{7 . 7} \pm \mathbf{2 . 7} * *$ & $\mathbf{4 . 4} \pm \mathbf{1 . 7}^{*}$ & $2.6 \pm 0.5$ & $2.2 \pm 0.3$ & $4.9 \pm 1.9$ & $3.2 \pm 1.4$ & $3.5 \pm 1.2$ \\
\hline ALP $(\mu \mathrm{kat} / \mathrm{l})$ & $2.3 \pm 0.4$ & $\mathbf{5 . 1} \pm \mathbf{1 . 8}^{*}$ & $\mathbf{5 . 3} \pm \mathbf{2 . 2 ^ { * }}$ & $4.0 \pm 0.8$ & $2.5 \pm 0.4$ & $4.8 \pm 1.0$ & $\mathbf{5 . 3} \pm \mathbf{1 . 8}^{\#}$ & $\mathbf{6 . 9}^{*} \pm \mathbf{1 . 2}^{\# \#, \mathbf{s}}$ \\
\hline $\begin{array}{l}\text { Total bilirubin } \\
(\mu \mathrm{mol} / \mathrm{l})\end{array}$ & $1.8 \pm 0.4$ & $6.8 \pm 5.0$ & $7.3 \pm 6.1$ & $5.7 \pm 1.6$ & $1.8 \pm 1.0$ & $5.2 \pm 1.2$ & $5.3 \pm 2.9$ & $\mathbf{6 . 0} \pm \mathbf{2 . 4 ^ { \# }}$ \\
\hline
\end{tabular}

The values represent the mean \pm SD $(n=6)$. ALT - alanine aminotransferase, AST - aspartate aminotransferase, ALP - alkaline phosphatase, HFD - high fat diet, PHx - partial hepatectomy, ST-1 - standard diet. * p $<0.05,{ }^{* *} \mathrm{p}<0.001$ vs ST-1; ${ }^{*} \mathrm{p}<0.05$, $\# \mathrm{p}<0.001$ vs HFD; ${ }^{\$} \mathrm{p}<0.05$ vs corresponding ST-1 group. 
ST-1 and HFD groups $(\mathrm{p}<0.001)$ with subsequent gradual decrease in BrdU incorporation $(48 \mathrm{~h}, \mathrm{p}<0.001 ; 72 \mathrm{~h}$ $\mathrm{p}<0.001)$. There were not differences between ST-1 and HFD group in DNA synthesis after PHx in any corresponding time interval. Nevertheless, zonal distribution of DNA synthesis was more pronounced in HFD groups lacking labeled cells in centrilobular zone.

\section{Liver characteristics}

Feeding with HFD induced significant accumulation of TAG $(\mathrm{p}<0.05)$ and cholesterol $(\mathrm{p}<0.001)$ in the liver (table 2). Accumulation of fat was confirmed by histological findings (oil red staining, data not shown). Although we did not observe inflammatory infiltration in the liver, fatty liver exerted increased markers of oxidative stress. Hepatic amount of GSH was lowered $(\mathrm{p}<0.05)$ and MDA content was increased $(\mathrm{p}<0.001)$ when compared with non-steatotic controls (table 2). Other liver parameters (absolute and relative liver weights, IL- 6 , TGF- $\beta 1$, content of DNA) did not significantly differ between ST-1 and HFD control groups.

Partial hepatectomy led to a significant decrease in absolute and relative liver weights, total and relative DNA contents in non-fatty and steatotic livers. In ST-1 group, PHx induced significant accumulation of TAG in the liver after 24 hours $(\mathrm{p}<0.05)$, whereas in HFD, PHx did not lead to additional increase in hepatic content of TAG. Hepatic cholesterol was significantly decreased 24, 48 and 72 hours after PHx only in HFD rats.

Regenerating liver exerted lowering of markers of oxidative stress when compared to corresponding controls.
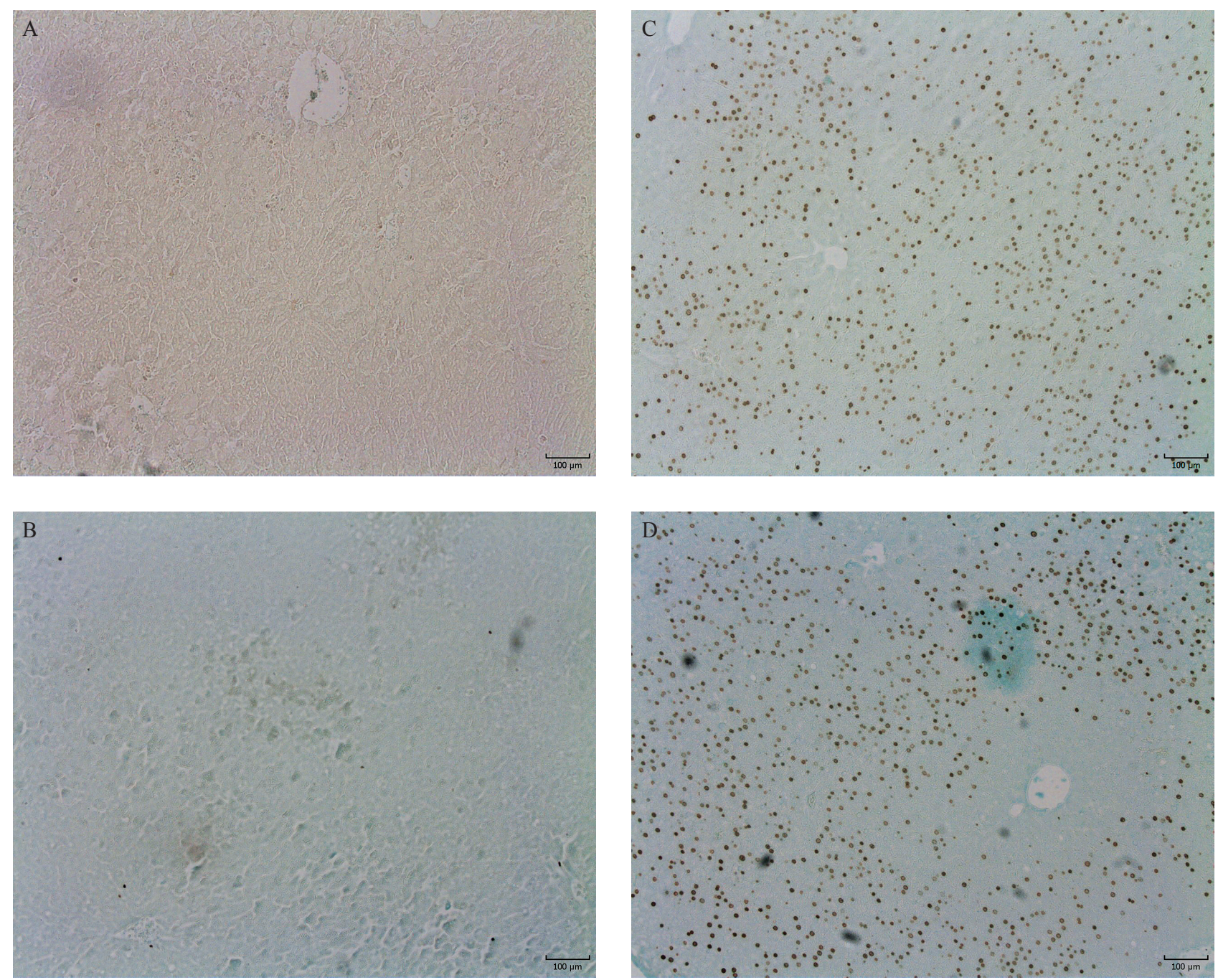

Fig. 1: Immunohistochemistry of BrdU incorporation in the liver. Samples of livers taken from control rats fed with ST-1 diet (1A) or HFD (1B) and from ST-1 (1C) and HFD (1D) animals 24 hours after PHx (ST-1 - control, HFD - high fat diet, PHx - partial hepatectomy). Objective magnification $10 \times$, bar $100 \mu \mathrm{m}$. 
Tab. 2: Basal liver characteristics of the groups.

\begin{tabular}{|c|c|c|c|c|c|c|c|c|}
\hline $\begin{array}{l}\text { Liver } \\
\text { characteristics }\end{array}$ & ST-1 & $\begin{array}{c}\text { ST-1_PHx } \\
24 \mathrm{~h}\end{array}$ & $\begin{array}{c}\text { ST-1_PHx } \\
48 \mathrm{~h}\end{array}$ & $\begin{array}{c}\text { ST-1_PHx } \\
72 \mathrm{~h}\end{array}$ & HFD & $\begin{array}{c}\text { HFD_PHx } \\
24 \mathrm{~h}\end{array}$ & $\begin{array}{c}\text { HFD_PHx } \\
48 \mathrm{~h}\end{array}$ & $\underset{72 \mathrm{~h}}{\text { HFD_PHx }}$ \\
\hline $\begin{array}{l}\text { Absolute liver } \\
\text { weight }(\mathrm{g})\end{array}$ & $11.0 \pm 1.2$ & $5.7 \pm 0.7^{* *}$ & $6.5 \pm 0.7$ & $7.2 \pm 0.5$ & $12.0 \pm 2.5$ & $5.2 \pm 0.5^{\ldots \# \#}$ & $7.5 \pm 1.5$ & $7.3 \pm 1.0$ \\
\hline $\begin{array}{l}\text { Relative liver } \\
\text { weight (\% of } \\
\text { body weight) }\end{array}$ & $2.5 \pm 0.1$ & $1.3 \pm 0.1 * * *$ & $1.6 \pm 0.1$ & $1.8 \pm 0.1$ & $2.6 \pm 0.2$ & $1.3 \pm 0.1^{\# \# \#}$ & $1.7 \pm 0.2$ & $1.9 \pm 0.1$ \\
\hline $\begin{array}{l}\text { Liver triacylgly- } \\
\text { cerols (mmol } / \mathrm{kg})\end{array}$ & $7.0 \pm 3.9$ & $50.0 \pm 21.9^{*}$ & $28.0 \pm 8.2$ & $26.2 \pm 9.2$ & $48.3 \pm 20.0^{*}$ & $53.6 \pm 18.5$ & $60.4 \pm 10.1$ & $53.6 \pm 9.7$ \\
\hline $\begin{array}{l}\text { Liver cholesterol } \\
(\mathrm{mmol} / \mathrm{kg})\end{array}$ & $7.1 \pm 2.0$ & $9.0 \pm 2.6$ & $7.8 \pm 0.9$ & $8.2 \pm 1.1$ & $31.8 \pm 8.7 * * *$ & $19.0 \pm 6.6^{\# \#, \mathrm{~s}}$ & $20.4 \pm 4.9^{\# \#, \mathrm{ss} s}$ & $15.1 \pm 3.1^{\# \# \#}$ \\
\hline $\begin{array}{l}\text { Liver GSH } \\
(\mathrm{mmol} / \mathrm{kg})\end{array}$ & $15.1 \pm 2.8$ & $21.6 \pm 4.1 * *$ & $19.0 \pm 2.1$ & $17.4 \pm 2.5$ & $9.6 \pm 3.6 *$ & $16.6 \pm 2.0^{\# \#}$ & $14.6 \pm 2.8$ & $18.6 \pm 1.0^{\ldots \#}$ \\
\hline $\begin{array}{l}\mathrm{GSH} / \mathrm{GSSG} \\
\text { ratio }\end{array}$ & $9.4 \pm 0.8$ & $10.8 \pm 0.8^{*}$ & $10.7 \pm 0.4 *$ & $11.4 \pm 0.7 * * *$ & $9.3 \pm 1.0$ & $10.0 \pm 0.8$ & $10.9 \pm 0.5^{\#}$ & $12.8 \pm 0.4^{\# \#, \mathrm{~s}}$ \\
\hline $\begin{array}{l}\text { Liver MDA } \\
\text { (nmol/g liver) }\end{array}$ & $28.2 \pm 2.8$ & $18.6 \pm 5.3$ & $11.1 \pm 6.2$ & $22.6 \pm 1.9$ & $72.1 \pm 17.9 * * *$ & $30.9 \pm 14.0^{\# \#}$ & $36.2 \pm 11.8^{\# \#, s s}$ & $47.5 \pm 11.0^{\# \#, s \$}$ \\
\hline $\begin{array}{l}\text { Liver IL-6 } \\
\text { (pg/mg protein) }\end{array}$ & $14.6 \pm 6.6$ & $20.7 \pm 2.0$ & $20.4 \pm 6.6$ & N/A & $16.1 \pm 4.6$ & $25.3 \pm 2.4 \#$ & $20.8 \pm 3.9$ & N/A \\
\hline $\begin{array}{l}\text { Liver TGF- } \beta 1 \\
\text { (pg/mg protein) }\end{array}$ & $15.7 \pm 2.7$ & $10.3 \pm 1.3$ & $10.6 \pm 3.7$ & $17.1 \pm 5.6$ & $16.3 \pm 6.9$ & $10.3 \pm 3.2$ & $10.7 \pm 2.5$ & $18.7 \pm 5.0$ \\
\hline $\begin{array}{l}\text { DNA content } \\
\text { (mg DNA/g } \\
\text { liver) }\end{array}$ & $1.7 \pm 0.1$ & $1.2 \pm 0.1^{*}$ & $1.2 \pm 0.4$ & $1.7 \pm 0.1$ & $1.7 \pm 0.1$ & $1.2 \pm 0.2$ & $1.5 \pm 0.6$ & $1.5 \pm 0.2$ \\
\hline $\begin{array}{l}\text { Total DNA } \\
\text { content (mg } \\
\text { DNA/liver) }\end{array}$ & $18.6 \pm 1.5$ & $6.8 \pm 0.9 * * *$ & $9.2 \pm 3.2$ & $12.5 \pm 1.1$ & $18.7 \pm 1.8$ & $6.0 \pm 0.8^{\# \#}$ & $9.2 \pm 1.9$ & $11.2 \pm 2.1$ \\
\hline
\end{tabular}

The values represent the mean \pm SD $(n=6)$. GSH - reduced form of glutathione, GSSG - oxidized form of glutathione, HFD - high fat diet, IL-6 - interleukin-6, MDA - malondialdehyde, PHx - partial hepatectomy, ST-1 - standard diet, TGF- $\beta 1$ - transforming growth factor $\beta 1{ }^{*} \mathrm{p}<0.05, * * \mathrm{p}<0.01, * * * \mathrm{p}<0.001$ vs ST-1; \# $\mathrm{p}<0.05$, \#\# $\mathrm{p}<0.01$, \#\#\# $\mathrm{p}<0.001$ vs HFD; $\$ \mathrm{p}<0.05, \$ \$$ $\mathrm{p}<0.01, \$ \$ \$ \mathrm{p}<0.001$ vs corresponding ST-1 group.

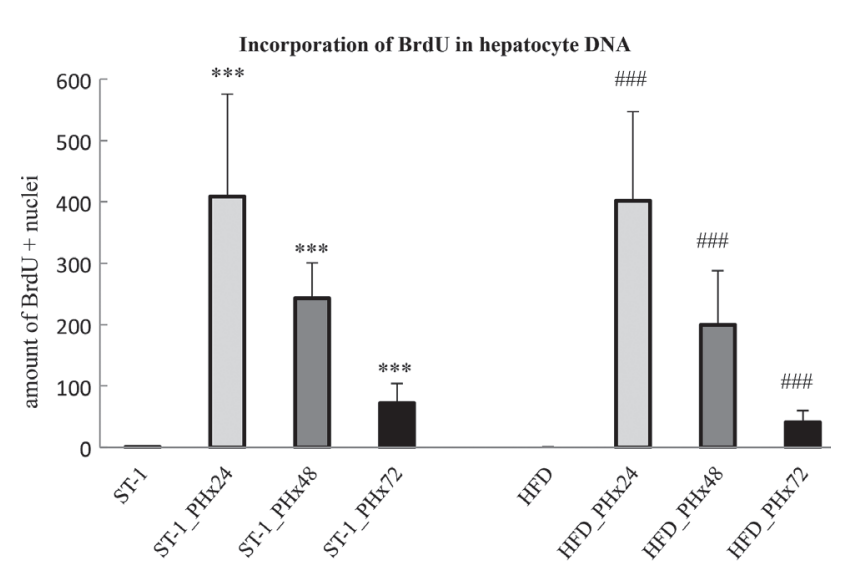

Fig. 2: Quantification of BrdU-positive nuclei in histopathological liver samples of ST-1 and HFD fed animals in 24, 48 and 72 hours after PHx. HFD - high fat diet, PHx - partial hepatectomy, ST-1 standard diet. $* * * \mathrm{p}<0.001$ vs ST-1; \#\#\# $\mathrm{p}<0.001$ vs HFD.
Liver GSH was elevated 24 hours after PHx in ST-1 animals $(\mathrm{p}<0.01)$ and $24(\mathrm{p}<0.01)$ and $72(\mathrm{p}<0.001)$ hours after PHx in HFD rats. Ratio of GSH to GSSG was increased in 24, 48 and 72 hours after PHx in ST-1 groups, and in 48 and $72 \mathrm{~h}$ in HFD. Hepatic MDA content was significantly decreased in all measured time intervals after PHx in HFD groups. A non-significant trend of liver MDA reduction after PHx was also observed in ST-1 groups.

An elevation of hepatic IL-6 level was induced by PHx in $24 \mathrm{~h}$ interval in HFD $(\mathrm{p}<0.05)$. A similar, non-significant increase in IL-6 was observed $24 \mathrm{~h}$ after PHx in ST-1 group. Liver content of TGB- $\beta 1$ exerted a non-significant decrease 24 and $48 \mathrm{~h}$ after PHx in both ST-1 and HFD groups.

\section{Respiration of mitochondria in liver homogenate}

As shown in table 3, respiration of mitochondria in liver homogenate at state 3 showed only a non-significant trend of increased oxygen consumption after addition of substrates of 
Tab. 3: Oxygen consumption of mitochondria in liver homogenate at state 3 and RCI.

\begin{tabular}{|l|c|c|c|c|c|c|c|c|}
\hline Respiration & ST-1 & $\begin{array}{c}\text { ST-1_PHx } \\
\mathbf{2 4} \mathbf{h}\end{array}$ & $\begin{array}{c}\text { ST-1_PHx } \\
\mathbf{4 8} \mathbf{h}\end{array}$ & $\begin{array}{c}\text { ST-1_PHx } \\
\mathbf{7 2 h}\end{array}$ & HFD & $\begin{array}{c}\text { HFD_PHx } \\
\mathbf{2 4} \mathbf{h}\end{array}$ & $\begin{array}{c}\text { HFD_PHx } \\
\mathbf{4 8 h}\end{array}$ & $\begin{array}{c}\text { HFD_PHx } \\
\mathbf{7 2 h}\end{array}$ \\
\hline $\begin{array}{l}\text { State 3 oxygen } \\
\text { consumption } \\
\text { at presence of } \\
\text { complex I sub- } \\
\text { strates }\end{array}$ & $100.0 \pm 23.7$ & $145.0 \pm 28.8$ & $167.6 \pm 58.7$ & $91.4 \pm 18.1$ & $92.1 \pm 36.6$ & $160.6 \pm 36.5$ & $156.5 \pm 19.9$ & $58.4 \pm 9.0$ \\
\hline $\begin{array}{l}\text { State 3 oxygen } \\
\text { consumption } \\
\text { at presence } \\
\text { of complex II } \\
\text { substrate }\end{array}$ & $100.0 \pm 24.9$ & $104.9 \pm 19.0$ & $112.4 \pm 33.2$ & $85.0 \pm 13.1$ & $102.3 \pm 2.4$ & $103.7 \pm 29.9$ & $112.8 \pm 30.6$ & $100.3 \pm 13.0$ \\
\hline $\begin{array}{l}\text { RCI at presence } \\
\text { of complex II } \\
\text { substrate }\end{array}$ & $2.9 \pm 0.6$ & $5.5 \pm 1.1$ & $3.2 \pm 0.3$ & $2.1 \pm 0.3$ & $4.0 \pm 0.6$ & $5.8 \pm 0.7$ & $4.5 \pm 1.2$ & $2.3 \pm 0.2$ \\
\hline
\end{tabular}

The values of respiration at state 3 represent the mean \pm SD and are expressed in $\%$ where $100 \%$ is respiration of ST- 1 control group. HFD - high fat diet, PHx - partial hepatectomy, RCI - respiratory control index, ST-1 - standard diet. n $=3-4$.

respiratory complex I in both ST-1 and HFD groups 24 and 48 hours after PHx. There were not changes in respiration at state 3 of complex II PHx in any group. RCI of complex II showed a non-significant trend of an elevation 24 hours after PHx in both groups.

\section{Discussion}

NAFLD is a frequent chronic liver disease and its worldwide prevalence continues to grow with the increasing incidence of obesity. The prevalence of NAFLD in Western countries is $20-30 \%$; about $2-3 \%$ of the general population suffers from non-alcoholic steatohepatitis (38). Steatosis is taken as an important risk factor for postoperative complication after major hepatectomy in experimental conditions and in clinical practice $(39,40)$.

Nevertheless, there are controversial data concerning the course of regeneration of steatotic liver; some authors found impaired regeneration of the liver affected by NAFLD (41, $42)$ while others did not $(25,43)$. The reasons of such inconsistency could be explained by different models, various degrees of steatosis and diverse stages of NAFLD used for induction of steatosis. Most of earlier studies were based on genetic leptin mutations (ob/ob mice, Zucker rats) or on methionine- and choline-deficient diet which do not correspond well with the picture of simple steatosis in human.

Therefore we decided to use nutritional model of steatosis which we described in our previous paper (14). In present study feeding rats with HFD for six weeks caused simple microvesicular steatosis without inflammatory reaction or fibrosis. Liver steatosis was confirmed by the triacylglycerol (TAG) content in the liver, which was almost sevenfold greater in HFD group in comparison to control rats. It is commonly known that the transient hepatocellular fat accu- mulation in the early phase of regeneration following $\mathrm{PHx}$ is required for physiological liver regeneration (44). Significantly increased TAG content in the liver 24 hours after PHx in rats fed with ST-1 is in good accordance with previous studies. In contrast to ST-1 fed animals, we did not observe any further changes in hepatic levels of TAG induced by partial hepatectomy in HFD rats.

Cell turnover in normal liver is very low and rate of hepatocyte DNA synthesis represents less than $0.1 \%$. It is generally accepted that homeostatic liver renewal arises by replication of pre-existing hepatocytes rather than stem cell differentiation (45). It was repeatedly documented using $\left[{ }^{3} \mathrm{H}\right]$-thymidine incorporation that the first hepatocytes to divide after PHx are periportal with the peak of DNA synthesis 18-20 hours after surgery $(46,47)$; DNA synthesis in centrilobular area is delayed by about 10 hours. BrdU labelling, widely used as a marker of proliferating hepatocytes, correlates well with the use of $\left[{ }^{3} \mathrm{H}\right]$-thymidine (48). Our results of significant increase of BrdU incorporation after PHx in control rats is in good concordance with findings of others $(1,46,47,49)$. Proliferative response in rats with simple steatosis was not altered in comparison to controls. The only difference was more pronounced zonal distribution of labelled hepatocytes with almost lacking cells in centrilobular zone in HFD rats. This could be explained by delayed onset of regenerative response as result from zonal kinetics of hepatocyte proliferation. Centrilobular zone has the poorest oxygen supply and the lack of oxygen can be further potentiated by high-fat diet in this zone (13). In accordance with our results Vetelainen and co-workers have shown that mild steatosis induced by a methionine- and choline-deficient diet did not affect liver regeneration after PHx; however, mild steatosis impaired functional recovery and increased hepatocellular damage after liver resection (21). Interleukin-6 
(IL-6) has been shown to play an important role in initiating liver regeneration via activating signal transducer and activator of transcription 3 (STAT3). The role of IL-6 dependent signalling during liver regeneration is attributed mainly to induction of acute phase response; serum levels of IL-6 in rats are elevated during the first hours after $\mathrm{PHx}$ preceding by hours increase of liver DNA synthesis (50). We found a trend of increasing liver IL-6 concentration after PHx in both control and HFD groups. Transforming growth factor beta1 (TGF- $\beta 1$ ) signalling pathway exerts an antiproliferative effect on hepatocytes. It has been documented that TGF- $\beta 1$ reversibly inhibits proliferative response after $\mathrm{PHx}$ and its signalling is inhibited in the early phase of liver regeneration (51). Our results fit their findings since we observed transient decrease of hepatic TGF- $\beta 1$ content 24 and 48 hours after PHx in both groups.

Impaired redox balance and oxidative stress belong to critical mechanisms in the pathogenesis of NAFLD (52). In accordance with this, we found that feeding with HFD caused induction of hepatic oxidative stress as documented by decreased content of GSH and increased concentration of MDA in steatotic liver. Partial hepatectomy attenuated level of lipoperoxidation and elevated content of GSH in steatotic liver. Similar effect was also observed in ST-1 fed rats. The increase in hepatic GSH after PHx corresponds to previous observations of Riehle and Huang $(53,54)$ who concluded that GSH is required for normal course of liver regeneration. An increase in GSH in the regenerating liver is necessary for hepatocyte entering the $\mathrm{S}$ phase (54). Moreover, mice deficient in GSH synthesis have also impaired priming, delayed DNA synthesis and low level apoptosis after PHx (53). Although we did not observe any significant difference in BrdU labelling after PHx in steatotic livers, the potential delay in $\mathrm{S}$ phase in these livers (absence of BrdU staining in pericentral area 24 hours after PHx in fatty livers) could be caused by significantly lower GSH content. Nevertheless, fatty livers maintain ability to increase hepatic level of $\mathrm{GSH}$ in response to liver resection which may explain no difference in DNA synthesis between steatotic and non-fatty livers after PHx. An increase of GSH during regeneration corresponds to a decrease in lipid peroxidation in both fatty and non-steatotic livers.

Mitochondrial dysfunction plays an important role in the development of NAFLD (12). Moreover, increased mitochondrial production of ROS may participate on impaired redox balance in NAFLD. Although many authors proofed changes in the function of mitochondria in NAFLD $(12,19$, $20,55)$, we did not observe significant differences in RCI for complex II and in state 3 respirations in the presence of complex I or II substrates between ST-1 and HFD. PHx did not induce any significant changes in respiration in any group. However, oxygen consumption at state 3 in the presence of complex I substrates showed a trend of transient increasing respiration 24 and 48 hours after PHx in both lean and steatotic groups. Such increase in respiration at state 3 after PHx was not detected when complex II substrate was used which corresponds with findings of Yang (56). We also observed a trend of transient increase in RCI at presence of complex II substrate in both groups $24 \mathrm{~h}$ after $\mathrm{PHx}$ which is in accordance with literature (56).

In summary, 2/3 partial hepatectomy-induced regeneration of the rat liver with simple microvesicular steatosis was not significantly affected when compared to the lean liver.

\section{Acknowledgements}

This study was supported by PRVOUK P37/02 and SVV 260287.

\section{References}

1. Bucher NL, Swaffield MN. The rate of incorporation of labelled thymidine into the deoxyribonucleic acid of regenerating rat liver in relation to the amount of liver excised. Cancer Res 1964; 24: 1611-25.

2. Michalopoulos GK, DeFrances MC. Liver regeneration. Science 1997; 276: 60-6.

3. Higgins G, Anderson R. Experimental pathology of the liver. I. Restoration of the liver of the white rat following partial surgical removal. Archives of pathology (Chic) 1931; 12: 186-202.

4. Fausto N. Liver regeneration. J Hepatol 2000; 32: 19-31.

5. Fausto N, Campbell JS, Riehle KJ. Liver regeneration. Hepatology 2006; 43 : S45-53.

6. Mangnall D, Bird NC, Majeed AW. The molecular physiology of liver regeneration following partial hepatectomy. Liver Int 2003; 23: 124-38.

7. Rychtrmoc D, Hubalkova L, Viskova A, Libra A, Buncek M, Cervinkova Z. Transcriptome temporal and functional analysis of liver regeneration termination. Physiol Res 2012; 61 Suppl 2: S77-92.

8. Trevisani F, Colantoni A, Caraceni P, Van Thiel DH. The use of donor fatty liver for liver transplantation: a challenge or a quagmire? J Hepatol 1996; 24: 114-21.

9. Behrns KE, Tsiotos GG, DeSouza NF, Krishna MK, Ludwig J, Nagorney DM. Hepatic steatosis as a potential risk factor for major hepatic resection. J Gastrointest Surg 1998; 2: 292-8.

10. Erickson SK. Nonalcoholic fatty liver disease. J Lipid Res 2009; 50 Suppl: S412-6.

11. Chalasani N, Younossi Z, Lavine JE, et al. The diagnosis and management of nonalcoholic fatty liver disease: Practice guideline by the American Association for the Study of Liver Diseases, American College of Gastroenterology, and the American Gastroenterological Association. Am J Gastroenterol 2012; 107: 811-26.

12. Vendemiale G, Grattagliano I, Caraceni P, et al. Mitochondrial oxidative injury and energy metabolism alteration in rat fatty liver: effect of the nutritional status. Hepatology 2001; 33: 808-15.

13. Mantena SK, Vaughn DP, Andringa KK, et al. High fat diet induces dysregulation of hepatic oxygen gradients and mitochondrial function in vivo. Biochem J 2009; 417: 183-93.

14. Kucera $\mathrm{O}$, Garnol T, Lotkova $\mathrm{H}$, et al. The effect of rat strain, diet composition and feeding period on the development of a nutritional model of non-alcoholic fatty liver disease in rats. Physiol Res 2011; 60: 317-28.

15. Paradies G, Paradies V, Ruggiero FM, Petrosillo G. Oxidative stress, cardiolipin and mitochondrial dysfunction in nonalcoholic fatty liver disease. World J Gastroenterol 2014; 20: 14205-18

16. Sanyal AJ, Campbell-Sargent C, Mirshahi F, et al. Nonalcoholic steatohepatitis: association of insulin resistance and mitochondrial abnormalities. Gastroenterology 2001; 120: 1183-92.

17. Kawahara H, Fukura M, Tsuchishima M, Takase S. Mutation of mitochondrial DNA in livers from patients with alcoholic hepatitis and nonalcoholic steatohepatitis. Alcohol Clin Exp Res 2007; 31: S54-60.

18. Gao D, Wei C, Chen L, Huang J, Yang S, Diehl AM. Oxidative DNA damage and DNA repair enzyme expression are inversely related in murine models of fatty liver disease. Am J Physiol Gastrointest Liver Physiol 2004; 287: G1070-7.

19. Perez-Carreras M, Del Hoyo P, Martin MA, et al. Defective hepatic mitochondrial respiratory chain in patients with nonalcoholic steatohepatitis. Hepatology 2003; 38: 999-1007.

20. Garcia-Ruiz I, Rodriguez-Juan C, Diaz-Sanjuan T, et al. Uric acid and anti-TNF antibody improve mitochondrial dysfunction in ob/ob mice. Hepatology 2006; 44 : 581-91.

21. Vetelainen R, Bennink RJ, van Vliet AK, van Gulik TM. Mild steatosis impairs functional recovery after liver resection in an experimental model. Br J Surg 2007; 94: 1002-8.

22. Selzner M, Clavien PA. Failure of regeneration of the steatotic rat liver: disruption at two different levels in the regeneration pathway. Hepatology 2000; 31: $35-42$. 
23. Kele PG, van der Jagt EJ, Gouw AS, Lisman T, Porte RJ, de Boer MT. The impact of hepatic steatosis on liver regeneration after partial hepatectomy. Liver Int 2013; 33: 469-75.

24. de Meijer VE, Kalish BT, Puder M, Ijzermans JN. Systematic review and metaanalysis of steatosis as a risk factor in major hepatic resection. Br J Surg 2010; 97 1331-9.

25. Picard C, Lambotte L, Starkel P, et al. Steatosis is not sufficient to cause an impaired regenerative response after partial hepatectomy in rats. J Hepatol 2002; 36: 645-52.

26. Sydor S, Gu Y, Schlattjan M, et al. Steatosis does not impair liver regeneration after partial hepatectomy. Lab Invest 2013; 93: 20-30.

27. Cho JY, Suh KS, Kwon CH, Yi NJ, Lee KU. Mild hepatic steatosis is not a major risk factor for hepatectomy and regenerative power is not impaired. Surgery 2006; 139: 508-15.

28. Lieber CS, Leo MA, Mak KM, et al. Model of nonalcoholic steatohepatitis. Am J Clin Nutr 2004; 79: 502-9.

29. Drahota Z, Palenickova E, Endlicher R, et al. Biguanides inhibit complex I, II and IV of rat liver mitochondria and modify their functional properties. Physiol Res 2014; 63: 1-11.

30. Cervinkova Z, Kucera O, Lotkova H, Drahota Z, Houstek J. [Oxygraphic evaluation of energy metabolism in isolated hepatocytes]. Acta Medica (Hradec Kralove) Suppl 2002; 45: 65-76.

31. Hissin PJ, Hilf R. A fluorometric method for determination of oxidized and reduced glutathione in tissues. Analytical biochemistry 1976; 74: 214-26.

32. Rousar T, Kucera O, Lotkova H, Cervinkova Z. Assessment of reduced glutathione: comparison of an optimized fluorometric assay with enzymatic recycling method. Anal Biochem 2012; 423: 236-40

33. Bligh EG, Dyer WJ. A rapid method of total lipid extraction and purification. Can J Biochem Physiol 1959; 37: 911-7.

34. Burton K. A study of the conditions and mechanism of the diphenylamine reaction for the colorimetric estimation of deoxyribonucleic acid. Biochem J 1956; 62: 315-23.

35. Bradford MM. A rapid and sensitive method for the quantitation of microgram quantities of protein utilizing the principle of protein-dye binding. Anal Biochem 1976; 72: 248-54

36. Pilz J, Meineke I, Gleiter CH. Measurement of free and bound malondialdehyde in plasma by high-performance liquid chromatography as the 2,4-dinitrophenylhydrazine derivative. J Chromatogr B Biomed Sci Appl 2000; 742: 315-25.

37. Bader A, Pavlica S, Deiwick A, et al. Proteomic analysis to display the effect of low doses of erythropoietin on rat liver regeneration. Life Sci 2011; 89: 827-33.

38. Bellentani S, Scaglioni F, Marino M, Bedogni G. Epidemiology of non-alcoholic fatty liver disease. Dig Dis 2010; 28: 155-61.

39. McCormack L, Petrowsky H, Jochum W, Furrer K, Clavien PA. Hepatic steatosis is a risk factor for postoperative complications after major hepatectomy: a matched case-control study. Ann Surg 2007; 245: 923-30.

40. Vetelainen R, van Vliet AK, van Gulik TM. Severe steatosis increases hepatocellular injury and impairs liver regeneration in a rat model of partial hepatectomy. Ann Surg. 2007; 245: 44-50.
41. Murata H, Yagi T, Iwagaki H, et al. Mechanism of impaired regeneration of fatty liver in mouse partial hepatectomy model. J Gastroenterol Hepatol 2007; 22: 2173-80.

42. DeAngelis RA, Markiewski MM, Taub R, Lambris JD. A high-fat diet impairs liver regeneration in C57BL/6 mice through overexpression of the NF-kappaB inhibitor, IkappaBalpha. Hepatology 2005; 42: 1148-57.

43. Rao MS, Papreddy K, Abecassis M, Hashimoto T. Regeneration of liver with marked fatty change following partial hepatectomy in rats. Dig Dis Sci 2001; 46: $1821-6$.

44. Rudnick DA, Davidson NO. Functional Relationships between Lipid Metabolism and Liver Regeneration. Int J Hepatol 2012; 2012: 549241.

45. Yanger K, Knigin D, Zong Y, et al. Adult hepatocytes are generated by self-duplication rather than stem cell differentiation. Cell Stem Cell 2014; 15: 340-9.

46. Fabrikant JI. The kinetics of cellular proliferation in regenerating liver. J Cell Biol 1968; 36: 551-65.

47. Grisham JW. A morphologic study of deoxyribonucleic acid synthesis and cell proliferation in regenerating rat liver; autoradiography with thymidine-H3. Cancer Res 1962; 22: 842-9.

48. Yu CC, Woods AL, Levison DA. The assessment of cellular proliferation by immunohistochemistry: a review of currently available methods and their applications. Histochem J 1992; 24: 121-31.

49. Weglarz TC, Sandgren EP. Timing of hepatocyte entry into DNA synthesis after partial hepatectomy is cell autonomous. Proc Natl Acad Sci U S A. 2000; 97 $12595-600$.

50. Streetz KL, Luedde T, Manns MP, Trautwein C. Interleukin 6 and liver regeneration. Gut 2000; 47: 309-12.

51. Russell WE, Coffey RJ, Jr., Ouellette AJ, Moses HL. Type beta transforming growth factor reversibly inhibits the early proliferative response to partial hepatectomy in the rat. Proc Natl Acad Sci U S A 1988; 85: 5126-30.

52. Gambino R, Musso G, Cassader M. Redox balance in the pathogenesis of nonalcoholic fatty liver disease: mechanisms and therapeutic opportunities. Antioxid Redox Signal 2011; 15: 1325-65.

53. Riehle KJ, Haque J, McMahan RS, Kavanagh TJ, Fausto N, Campbell JS. Sustained Glutathione Deficiency Interferes with the Liver Response to TNF-alpha and Liver Regeneration after Partial Hepatectomy in Mice. J Liver Disease Transplant 2013; 1 .

54. Huang ZZ, Li H, Cai J, Kuhlenkamp J, Kaplowitz N, Lu SC. Changes in glutathione homeostasis during liver regeneration in the rat. Hepatology 1998; 27: 147-53.

55. Cortez-Pinto H, Chatham J, Chacko VP, Arnold C, Rashid A, Diehl AM. Alterations in liver ATP homeostasis in human nonalcoholic steatohepatitis: a pilot study. JAMA 1999; 282: 1659-64.

56. Yang S, Tan TM, Wee A, Leow CK. Mitochondrial respiratory function and antioxidant capacity in normal and cirrhotic livers following partial hepatectomy. Cell Mol Life Sci 2004; 61: 220-9.

Received: 23/05/2016

Accepted: 06/06/2016 